\begin{tabular}{|c|l|}
\hline Title & On a lower bound for the extinction time of surfaces moved by mean curvature \\
\hline Author(s) & Giga, Y.; Y amauchi, K. \\
\hline Citation & Hokkaido University Preprint Series in Mathematics, 185, 1-16 \\
\hline Issue Date & 1993-03 \\
\hline DOI & 10.14943/83329 \\
\hline Doc URL & http://hdl.handle.net/2115/68931 \\
\hline Type & bulletin (article) \\
\hline File Information & pre185.pdf \\
\hline
\end{tabular}

Instructions for use 
On a Lower Bound for the Extinction Time of Surfaces Moved by Mean Curvature

Y. Giga and K. Yama-uchi

Series $\sharp 185$. March 1993 


\section{HOKKAIDO UNIVERSITY \\ PREPRINT SERIES IN MATHEMATICS}

$\sharp 157$ : A. Arai, Momentum operators with gauge potentials, local quantization of magnetic $f$ ux, and representation of canonical commutation relations, 11 pages. 1992.

$\sharp 158$ : S. Izumiya, W.L. Marar, The Euler number of a topologically stable singular surface in a 3-manifold, 11 pages. 1992.

$\sharp 159: \quad$ T. Hibi, Cohen-Macaulay types of Cohen-Macaulay complexes, 26 pages. 1992.

$\sharp$ 160: A. Arai, Properties of the Dirac-Weyl operator with a strongly singular gauge potential, 26 pages. 1992.

\#161: A. Arai, Dirac operators in Boson-Fermion Fock spaces and supersymmetric quantum field theory, 30 pages. 1992.

$\sharp 162$ : S. Albeverio, K. Iwata, T. Kolsrud, Random parallel transport on surfaces of finite type, and relations to homotopy, 8 pages. 1992.

\#163: S. Albeverio, K. Iwata, T. Kolsrud, Moments of random fields over a family of elliptic curves, and modular forms, 9 pages. 1992.

$\sharp 164$ : Y. Giga, M. Sato, Neumann problem for singular degenerate parabolic equations, 12 pages. 1992.

$\sharp 165$ : J. Wierzbicki, Y. Watatani, Commuting squares and relative entropy for two subfactors, 18 pages. 1992.

\#166: Y. Okabe, A new algorithm driven from the view-point of the fluctuation-dissipation theorem in the theory of $\mathrm{KM}_{2} \mathrm{O}$-Langevin equations, 13 pages. 1992.

$\sharp 167$ : Y. Okabe, H. Mano and Y. Itoh, Random collision model for interacting populations of two species and its strong law of large numbers, 14 pages. 1992.

$\sharp 168$ : A. Inoue, On the equations of stationary precesses with divergent diff usion coefficients, 25 pages. 1992.

$\sharp 169$ : T. Ozawa, Remarks on quadratic nonlinear Schrödinger equations, 19 pages. 1992.

$\$ 170$ : T. Fukui, Y. Giga, Motion of a graph by nonsmooth weighted curvature, 11 pages. 1992.

\#171: J. Inoue, T. Nakazi, Finite dimensional solution sets of extremal problems in $\mathrm{H}^{1}, 10$ pages. 1992.

$\sharp 172$ : S. Izumiya, A characterization of complete integrability for partial differential equations of first order, 6 pages. 1992.

$\sharp 173$ : T. Suwa, Unfoldings of codimension one complex analytic foliation singularities, 49 pages. 1992.

$\sharp 174$ : T. Ozawa, Wave propagation in even dimensional spaces, 15 pages. 1992.

$\sharp 175: \quad$ S. Izumiya, Systems of Clairaut type, 7 pages. 1992.

$\sharp 176$ : A. Hoshiga, The initial value problems for quasi-linear wave equations in two space dimensions with small data, 25 pages. 1992.

\#177: K. Sugano, On bicommutators of modules over H-separable extension rings III, 9 pages. 1993.

$\sharp 178$ : T. Nakazi, Toeplitz operators and weighted norm inequalities, 17 pages. 1993.

$\sharp 179: \quad$ O. Ogurisu, Existence and structure of infinitely degenerate zero-energy ground states of a Wess-Zumino type model in supersymmetric quantum mechanics, 26 pages. 1993.

$\sharp 180$ : O. Ogurisu, Ground state of a spin 1/2 charged particle in an even dimensional magnetic field, 9 pages. 1993.

$\sharp 181$ : K. Sugano, Note on H-separable Galois extension, 6 pages. 1993.

\#182: M. Yamada, Distance formulas of asymptotic Toeplitz and Hankel operators, 13 pages. 1993

\# 183: G. Ishikawa, T. Ozawa, The genus of a connected compact real algebraic surface in the affine three space, 11 pages. 1993.

$\sharp$ 184: T. Hibi, Canonical modules and Cohen-Macaulay types of partially ordered sets, 6 pages. 1993. 


\title{
On a Lower Bound for the Extinction Time of Surfaces Moved by Mean Curvature
}

\author{
Yoshikazu Giga* and Kazuyuki Yama-uchi
}

Department of Mathematics, Hokkaido University, Sapporo 060, Japan

\section{Introduction}

We consider a one-parameter family of bounded open sets $\left\{D_{t}\right\}_{t \geq 0}$ in $R^{n}(n \geq 2)$ whose boundary $\Gamma_{t}=\partial D_{t}$ is moving by its mean curvature. The initial value problem describing this motion is

$$
\left\{\begin{array}{l}
V=H \quad \text { on } \quad \Gamma_{t} \\
\left.\Gamma_{t}\right|_{t=0}=\Gamma_{0}=\partial D_{0} .
\end{array}\right.
$$

Here $V=V(t, x)$ and $H=H(t, x)$ are, respectively, the inward normal velocity and the inward ( $n-1$ times) mean curvature of $\Gamma_{t}$ at a point $x$ of $\Gamma_{t}$.

By the level set method it is known that the problem (E) admits a unique global generalized solution $\left\{\Gamma_{t}\right\}$ (or $\left\{D_{t}\right\}$ ) provided that $D_{0}$ is a bounded open set [CGG1], [ES1]. (The paper [CGG3] includes corrections of technical errors in [CGG1]). It is also known that $D_{t}$ becomes empty in a finite time. For example if $D_{0}$ is an open ball $B^{n}(R)$ of radius $R$ (centered at the origin),

$$
D_{t}=B^{n}(R(t)) \quad \text { with } \quad R(t)^{2}=R^{2}-2(n-1) t
$$

for $t<t_{R}=R^{2} / 2(n-1)$ and $D_{t}$ is empty for $t \geq t_{R}$. For a general initial data $D_{0}$ we consider a large ball $B^{n}(R)$ containing $D_{0}$. Applying the comparison principle in [CGG1], [ES1], we now observe that $D_{t}$ becomes empty by the time $t=t_{R}$. Thus the extinction time defined by

$$
t_{*}=t_{*}\left(D_{0}\right)=\inf \left\{t ; D_{t}=\emptyset\right\}
$$

is finite since $t_{*} \leq t_{R}$ provided that $D_{0}$ is bounded; here $D_{t}$ is a unique generalized solution of $(E)$.

\footnotetext{
* Partly supported by the Inamori Foundation
} 
As for upper bounds of $t_{*}$, besides the trivial estimate $t_{*} \leq t_{R}$ L.C. Evans and J. Spruck [ES3] proved an interesting estimate

$$
t_{*} \leq C \mathscr{H}^{n-1}\left(\Gamma_{0}\right)^{2 /(n-1)}
$$

with some constant $C$ depending only on $n$. Here $\mathscr{H}^{n-1}$ denotes the $n-1$ dimensional Hausdorff measure. Actually, their estimate is still valid even if $t_{*}$ is replaced by the extinction time of $\Gamma_{t}$ which may be greater than $t_{*}$ because generalized interface evolution $\Gamma_{t}$ may fatten for nonsmooth $\Gamma_{0}[\mathrm{ES} 1]$.

Our goal in this note is to derive a lower bound of $t_{*}$ by geometric quantities of initial data. A trivial estimate for $t_{*}$ is obtained by comparison with balls contained in $D_{0}$. However, as far as the authors know, our estimate provides the first nontrivial lower bound for $t_{*}$. Let $\mathscr{L}^{n}$ denote the Lebesgue measure in $R^{n}$. Then our main result is

Theorem 1. Let $D_{0}$ be a smoothly bounded open set in $R^{n}$ and $t_{*}\left(D_{0}\right)$ be the extinction time defined in (1.1). Then $t_{*}=t_{*}\left(D_{0}\right)$ is estimated as

$$
t_{*} \geq 2\left(\mathscr{L}^{n}\left(D_{0}\right) / \mathscr{H}^{n-1}\left(\Gamma_{0}\right)\right)^{2}
$$

The constant 2 is optimal for this estimate.

Remark. We may weaken the assumption on the regularity of $\partial D_{0}$. As observed later, it suffices to assume that $\partial D_{0}$ is $n-1$ rectifiable in the sense of Federer $[F]$.

If $D_{0}=B^{n}(R)$, then the right hand side of (1.3) equals $2 R^{2} / n^{2}$. Since $t_{R}=R^{2} / 2(n-1)$, for $n=2$ the equality in (1.3) holds for $D_{0}=B^{2}(R)$. However $n \geq 3$ the estimate (1.3) is not sharp for $B^{n}(R)$. As discussed later, (1.3) is sharper than the trivial estimate if $D_{0}$ is a "thin bar" containing no large balls.

The estimate (1.3) is easily proved when $\left\{\Gamma_{t}\right\}_{0 \leq t<t_{*}}$ is a smooth family of smooth surfaces. Indeed, set

$$
\begin{array}{ll}
v(t)=\mathscr{L}^{n}\left(D_{t}\right) \quad\left(\text { the volume of } D_{t}\right) \\
\left.a(t)=\mathscr{H}^{n-1}\left(\Gamma_{t}\right) \quad \text { (the area of } \Gamma_{t}\right) .
\end{array}
$$


We calculate the time derivative of $a$ and $v$. Since $V=H$, we observe (see e.g. [H]) that

$$
\begin{aligned}
-a^{\prime}(t) & =\int V H d \mathscr{H}^{n-1}=\int H^{2} d \mathscr{H}^{n-1} \geq 0 \\
-v^{\prime}(t) & =\int V d \mathscr{H}^{n-1}=\int H d \mathscr{H}^{n-1},
\end{aligned}
$$

where the integrals are over $\Gamma_{t}$. Applying the Schwarz inequality now yields

$$
-v^{\prime}(t) \leq\left(\int 1 d \mathscr{H}^{n-1} \int H^{2} d \mathscr{H}^{n-1}\right)^{1 / 2}=a^{1 / 2}\left(-a^{\prime}\right)^{1 / 2}=\left(-\left(a^{2}\right)^{\prime} / 2\right)^{1 / 2} .
$$

Integrating this inequality over $\left(0, t_{*}\right)$ yields

$$
\begin{aligned}
v(0)-v\left(t_{*}\right) & \leq \int_{0}^{t_{*}}\left(-\left(a^{2}\right)^{\prime} / 2\right)^{1 / 2} d t \\
& \leq\left(\int_{0}^{t_{*}} 1 d t \int_{0}^{t_{*}}\left(-\left(a^{2}\right)^{\prime} / 2\right) d t\right)^{1 / 2}(\text { by the } \\
& =\left(t_{*} / 2\right)^{1 / 2}\left[a^{2}(0)-a^{2}\left(t_{*}\right)\right]^{1 / 2}
\end{aligned}
$$

Since $a\left(t_{*}\right)=v\left(t_{*}\right)=0$, this yields $(1.3)$.

For $n=2$ the equation (E) admits a unique smooth solution $\Gamma_{t}$ until it shrinks to a point at $t=t_{*}$. This follows from results of [Gr1] and [GH]. Since smooth solution $\left\{\Gamma_{t}\right\}$ is also a generalized solution (see [ES1]), the formal proof of (1.3) given in the preceeding paragraph is sufficient to show (1.3) provided $n=2$. Even if $n \geq 3$ the formal proof is sufficient if $D_{0}$ is convex, since $D_{t}$ stays smooth and convex until it disappears at time $t=t_{*}[\mathrm{H}]$.

Unfortunately, for $n \geq 3$ the surface $\left\{\Gamma_{t}\right\}$ may become singular at some time before $t_{*}$ even if the initial surface $\Gamma_{0}$ is smooth. In fact if $\Gamma_{0}$ is a barbell with thin neck, it pintches in the middle before it disappears, as shown in [Gr2]. To show (1.3) for generalized solutions we should modify the formal proof for solutions of the level set equation, where each level set of solutions moves by its mean curvature. This is a main technical part of this note.

The bibiography of $[A A G]$ includes many references to recent work on the generalized solution of the mean curvature flow equation $(E)$. We take the opportunity to note some other, related articles not cited there and not mentioned elsewhere in this introduction. In 
[I] Ilmanen proved that the singular limit of solution for the Allen -Cahn equation yields a Brakke's varifold solution to $(\mathrm{E})$ and that its support is contained in our generalized interface evolution. In [ES4] Evans and Spruck proved that the almost every set of solution for the level set equation (L) is a unit density varifold solution in the sense of Brakke [B].

\section{Extinction time for the level set equation}

We recall generalized solutions of (E) constructed by [ES1] and [CGG1]. For a bounded open set $D_{0}$ we take $g \in K_{\alpha}$ such that $D_{0}=\{g>0\}$, the super zero-level set of $g$. Here $\alpha$ is a positive number and $K_{\alpha}$ is the space of all continuous functions on $R^{n}$ whose values equal to $-\alpha$ outside some compact subset of $\boldsymbol{R}^{n}$. We then consider the level set equation for $(E)$ :

$$
\left\{\begin{array}{l}
u_{t}-|\nabla u| \operatorname{div}(\nabla u /|\nabla u|)=0 \quad \text { in }(0, \infty) \times R^{n} \\
\left.u\right|_{t=0}=g \text { on } R^{n}
\end{array}\right.
$$

The equation (L) admits a unique global solution in viscosity sense [CGG1], [ES1]. As stated in [AAG, Theorem 3.1] (see also [ES1] and [CGG1]) the super zero-level set of $u(t, \cdot)$, i.e.,

$$
D_{t}=\{x ; u(t, x)>0\}
$$

is independent of the choice of $g$ and depends only on $D_{0}$. The family $\left\{D_{t}\right\}_{t \geq 0}$ is called the generalized solution (or inner evolution) with initial data $D_{0}$. We shall state a lower bound of $t_{*}=t_{*}\left(D_{0}\right)$ in terms of $g$ which is crucial in proving (1.3). We set

$$
\int_{U}|\nabla g| \equiv \sup \left\{\int_{U} g \operatorname{div} \varphi d x ; \varphi \in C_{0}^{\infty}\left(U, R^{n}\right),|\varphi(x)| \leq 1\right\}
$$

which is the total variation of $\nabla g$ in an open set $U$ in $R^{n}$. If $g$ is smooth, $\int|\nabla g|=\int|\nabla g| d x$ where the integrals are over $U$.

Theorem 2. Assume that $g \in K_{\alpha}$ for some $\alpha>0$ such that $D_{0}$ is a super zero-level set of $g$. Let $t_{*}=t_{*}\left(D_{0}\right)$ be the extinction time defined by (1.1). Then

$$
2\left(\int_{D_{0}} g(x) d x\right)^{2} \leq t_{*}\left(\int_{D_{0}}|\nabla g|\right)^{2}
$$


Proof of (1.3) admitting Theorem 2. Let $d$ denote a signed distance function of $\partial D_{0}$ defined by

$$
d(x)= \begin{cases}\operatorname{dist}\left(x, \partial D_{0}\right), & \text { for } x \in D_{0} \\ -\operatorname{dist}\left(x, \partial D_{0}\right), & \text { otherwise }\end{cases}
$$

We approximate the signiture function by a Lipschitz function $\theta_{m}(m=1,2, \cdots)$ defined by

$$
\theta_{m}(\xi)= \begin{cases}1, & \text { if } \xi \geq 1 / m \\ m \xi, & \text { if }|\xi| \leq 1 / m \\ -1 & \text { if } \xi \leq-1 / m\end{cases}
$$

By definition the function $g_{m}=\theta_{m}(d)$ belongs to $K_{1}$ and the super zero-level set of $g_{m}$ agrees with $D_{0}$. Applying Theorem 2 yields

$$
2\left(\int_{D_{0}} g_{m} d x\right)^{2} \leq t_{*}\left(\int_{D_{0}}\left|\nabla g_{m}\right|\right)^{2}
$$

Letting $m \rightarrow \infty$ yields (1.3), if we prove

$$
\lim _{m \rightarrow \infty} \int_{D_{0}} g_{m} d x=\mathscr{L}^{n}\left(D_{0}\right), \quad \lim _{m \rightarrow \infty} \int_{D_{0}}\left|\nabla g_{m}\right|=\mathscr{H}^{n-1}\left(\partial D_{0}\right)
$$

The first convergence is clear from the monotone convergence theorem.

It remains to prove the last convergence. Since $d$ and $\theta_{m}$ are Lipschitz, so is $g_{m}$. Moreover, $\nabla g_{m}=\theta_{m}^{\prime}(d) \nabla d$ in the sense of distribution and $|\nabla d|=1$ almost everywhere. We thus observe that

$$
\int_{D_{0}}\left|\nabla g_{m}\right|=\int_{D_{0}}\left|\nabla g_{m}\right| d x=\int_{T_{m}} \theta_{m}^{\prime}(d)|\nabla d| d x=\int_{T_{m}} m \cdot 1 d x=m \mathscr{L}^{n}\left(T_{m}\right) .
$$

with $T_{m}=\left\{x \in D_{0} ; 0 \leq d(x) \leq 1 / m\right\}$. It now suffices to prove

$$
\lim _{m \rightarrow \infty} m \mathscr{L}^{n}\left(T_{m}\right)=\mathscr{H}^{n-1}\left(\partial D_{0}\right)
$$

which is easy to prove when $\partial D_{0}$ is smooth enough. According to $[F]$, this convergence is also valid even if $\partial D_{0}$ is merely rectifiable. Since this is the only part we use regularity of $\partial D_{0}$ we have proved (1.3) with the remark to Theorem 1.

Proof of the second assertion of Theorem 1. If $n=2$, the equality in (1.3) holds for $D_{0}=B^{2}(R)$. We shall assume $n \geq 3$ and prove that the constant 2 in (1.3) is optimal 
even if we restrict $D_{0}$ as a smooth, strictly convex, bounded open set. For each $\ell>1$ let $V_{\ell}$ denote

$$
V_{\ell}=\left\{x=(y, z) \in R^{n-2} \times R^{2} ;(|y| / \ell)^{2 \ell}+|z|^{2 \ell}<1\right\}
$$

Let us calculate the volume of $V_{\ell}$ by a change of variables:

$$
\begin{aligned}
\mathscr{L}^{n}\left(V_{\ell}\right) & =\int_{|y| \leq \ell}\left(\int_{0}^{2 \pi} \int_{0}^{s(|y|)} s d s d \theta\right) d y=\pi \int_{|y| \leq \ell} s^{2}(|y|) d y, y=\ell \eta \\
& =\pi \ell^{n-2} \int_{|\eta| \leq 1} \sigma^{2}(|\eta|) d \eta \quad \text { with } \quad \sigma(\rho)=\left(1-\rho^{2 \ell}\right)^{1 / 2 \ell}, s(r)=\sigma(r / \ell) .
\end{aligned}
$$

Since $\sigma(\rho) \geq 1-\rho^{2 \ell}$, we observe that

$$
\mathscr{L}^{n}\left(V_{\ell}\right) \geq \pi \ell^{n-2} \int_{|\eta| \leq 1}\left(1-|\eta|^{2 \ell}\right) d \eta=\pi \omega \ell^{n-2}(1-O(1 / \ell)) \quad \text { as } \quad \ell \rightarrow \infty
$$

where $\omega=\mathscr{L}^{n-2}\left(B^{n-2}(1)\right)$. Since each cross section of $V_{\ell}$ at $y=$ const. is a disk of radius $s(|y|)$, we see

$$
\begin{aligned}
\mathscr{H}^{n-1}\left(\partial V_{\ell}\right) & =2 \pi \int_{|y| \leq \ell} s(r)\left(1+\left|\nabla_{y} s(r)\right|^{2}\right)^{1 / 2} d y, & & r=|y| \\
& =2 \pi \ell^{n-2} \int_{|\eta| \leq 1} \sigma(\rho)\left(1+\left|\nabla_{\eta} \sigma(\rho)\right|^{2} / \ell^{2}\right)^{1 / 2} d \eta, & & \rho=|\eta|
\end{aligned}
$$

by a change of variable: $y=\ell \eta$. This yields the estimate

$$
\begin{aligned}
\mathscr{H}^{n-1}\left(\partial V_{\ell}\right) & \leq 2 \pi \ell^{n-2} \int_{|\eta| \leq 1}\left(\sigma(\rho)+\ell^{-1}\left|\nabla_{\eta} \sigma(\rho)\right| \sigma(\rho)\right) d \eta \\
& \leq 2 \pi \ell^{n-2}\left(\omega-\ell^{-1} \int_{|\eta| \leq 1} \sigma^{\prime}(\rho) \sigma(\rho) d \eta\right)
\end{aligned}
$$

since $\sigma(\rho) \leq 1$ for $|\rho| \leq 1$ and $\left|\nabla_{\eta} \sigma(\rho)\right|=-\sigma^{\prime}(\rho)$. Note that

$$
-2 \int_{0}^{1} \sigma^{\prime}(\rho) \sigma(\rho) \rho^{n-3} d \rho=-\int_{0}^{1}\left(\sigma^{2}(\rho)\right)^{\prime} \rho^{n-3} d \rho=-\left[\sigma^{2}(\rho) \rho^{n-3}\right]_{0}^{1}+\int_{0}^{1} \sigma^{2}(\rho)\left(\rho^{n-3}\right)^{\prime} d \rho
$$

is bounded for $\ell \geq 1$. We thus observe that

$$
\int_{|\eta| \leq 1} \sigma^{\prime}(\rho) \sigma(\rho) d \eta=O(1) \quad \text { as } \quad \ell \rightarrow \infty
$$


which now yields

$$
\mathscr{H}^{n-1}\left(\partial V_{\ell}\right) \leq 2 \pi \ell^{n-2}(\omega+O(1 / \ell)) \text { as } \ell \rightarrow \infty
$$

Combining the estimate of $\mathscr{L}^{n}\left(V_{\ell}\right)$ yields

$$
4\left(\mathscr{L}^{n}\left(V_{\ell}\right) / \mathscr{H}^{n-1}\left(V_{\ell}\right)\right)^{2} \geq 1+O(1 / \ell) \text { as } \ell \rightarrow \infty
$$

Let $t_{\ell}$ be the extinction time for $V_{\ell}$, i.e. $t_{\ell}=t_{*}\left(V_{\ell}\right)$. Since $V_{\ell}$ is smooth and convex and since $V_{\ell}$ is contained in a cylinder $R^{n-2} \times B^{2}(1)$, the classical comparison yields $t_{\ell} \leq 1 / 2$; note that $B^{2}(1)$ extincts at the time $1 / 2$. Comparing the estimate of $\left(\mathscr{L}^{n}\left(V_{\ell}\right) / \mathscr{H}^{n-1}\left(V_{\ell}\right)\right)^{2}$ we now observe that the constant 2 in (1.3) optimal by taking $\ell \rightarrow \infty$.

\section{Approximate level set equations}

The basic idea in proving Theorem 2 is found in the formal proof of (1.3) in Section 1. Although it is abuse of notation, we set

$$
V=-u_{t} /|\nabla u| \quad \text { and } \quad H=-\operatorname{div}(\nabla u /|\nabla u|)
$$

since they represent, respectively, the inward normal velocity and the inward mean curvatue on each level set of $u$. Suppose that $u$ is a solution of $(\mathrm{L})$. Then formally we have

$$
\begin{aligned}
& -\frac{d}{d t} \int|\nabla u| d x=-\int \frac{\nabla u_{t} \cdot \nabla u}{|\nabla u|} d x=-\int u_{t} H d x=-\int V H|\nabla u| d x=\int H^{2}|\nabla u| d x \\
& -\frac{d}{d t} \int u d x=-\int u_{t} d x=\int V|\nabla u| d x=\int H|\nabla u| d x
\end{aligned}
$$

by the integration by parts, where the integrals are $R^{n}$. Identities (3.1) and (3.2) are regarded as a level set formulation of (1.4) and (1.5), respectively and are still important to prove Theorem 2 at least conceptually. Unfortunately, since (L) is degenerate parabolic, we cannot expect that $u$ is differentiable so it is nontrivial to interpret (3.1) and (3.2)

To overcome this difficulty we consider the approximate level set equation for $\varepsilon>0$ :

$$
\left\{\begin{array}{l}
u_{t}-|\nabla u|_{\varepsilon} \operatorname{div} N^{\varepsilon}(\nabla u)=0 \quad \text { in }(0, \infty) \times R^{n} \\
\left.u\right|_{t=0}=g \text { on } R^{n}
\end{array}\right.
$$


with $|p|_{\varepsilon}=\left(\varepsilon^{2}+|p|^{2}\right)^{1 / 2}, N^{\varepsilon}(p)=p /|p|_{\varepsilon}$ for $p \in R^{n}$. This equation is studied in [ES1] to construct a solution of $(L)$ as a locally uniform limit of a solution of $\left(A_{\varepsilon}\right)$ as $\varepsilon \rightarrow 0$. In fact, the equation $\left(A_{\varepsilon}\right)$ admits a unique global solution $u^{\varepsilon}$ (smooth for $t>0$ and continuous for $t \geq 0$ ) for arbitrary $g \in K_{\alpha}$. Applying the maximum principle yields

$$
\sup _{0<\varepsilon<1}\left\{\left\|u^{\varepsilon}\right\|_{\infty},\left\|\nabla u^{\varepsilon}\right\|_{\infty},\left\|u_{t}^{\varepsilon}\right\|_{\infty}\right\} \leq C\|g\|_{C^{2}}
$$

for $g \in C^{2}$ with some constant $C=C(n)$ independent of $g$; here $\|f\|_{\infty}$ and $\|f\|_{C^{2}}$ denote, respectively, the maximum norm and $C^{2}$ norm of $f$. By the Ascoli-Arzela theorem $u^{\varepsilon}$ converges to some function $u$ locally uniformly by taking a subsequences. However since $u$ solves (L), the limit $u$ is unique so $u^{\varepsilon}$ itself converges to $u$. Even if $g$ is merely in $K_{\alpha}$ using the comparison principle for (L) ([ES1, CGG1]), we observe that

$$
u^{\varepsilon} \rightarrow u \text { locally uniform on }[0, \infty) \times R^{n} \text { as } \varepsilon \rightarrow 0
$$

for $u$ which solves (L). We refer to [ES1] for details.

We still have a problem to interpret (3.1) and (3.2) even if $u^{\varepsilon}$ and $\left|\nabla u^{\varepsilon}\right|_{\varepsilon}$ replace $u$ and $|\nabla u|$ respectively, since $\left|\nabla u^{\varepsilon}\right|_{\varepsilon}=\left(\varepsilon^{2}+\left|\nabla u^{\varepsilon}\right|^{2}\right)^{1 / 2}$ is not integrable on $R^{n}$. We consider integrals over a bounded set. Then we should estimate the boundary terms which comes from integration by parts.

Let $\theta$ be a nondecreasing $C^{2}$ function on $R$ and $\Omega$ be a smoothly bounded open set in $R^{n}$. We consider "approximate" area and volume functions:

$$
\begin{aligned}
& a^{\varepsilon}(t)=\int_{\Omega} \theta^{\prime}\left(u^{\varepsilon}\right)\left|\nabla u^{\varepsilon}\right|_{\varepsilon} d x, \quad|p|_{\varepsilon}=\left(\varepsilon^{2}+|p|^{2}\right)^{1 / 2} \\
& v^{\varepsilon}(t)=\int_{\Omega} \theta\left(u^{\varepsilon}\right) d x .
\end{aligned}
$$

To express error terms we set $U^{\varepsilon}=\theta\left(u^{\varepsilon}\right)$ and, for $0 \leq t_{0}<\infty$,

$$
\begin{array}{ll}
B^{\varepsilon}\left(t_{0}\right)=\int_{0}^{t_{0}} a^{\varepsilon}(t) b^{\varepsilon}(t) d t, & b^{\varepsilon}(t)=\int_{\partial \Omega} U_{t}^{\varepsilon} N^{\varepsilon}\left(\nabla u^{\varepsilon}\right) \cdot \nu d \mathscr{H}^{n-1} \\
E^{\varepsilon}\left(t_{0}\right)=\int_{0}^{t_{0}} a^{\varepsilon}(t) e^{\varepsilon}(t) d t, & e^{\varepsilon}(t)=\int_{\Omega}\left|\theta^{\prime \prime}\left(u^{\varepsilon}\right) u_{t}^{\varepsilon}\right| d x,
\end{array}
$$

where $\nu$ is the unit exterior normal vector of $\partial \Omega$ and $N^{\varepsilon}(p)=p /|p|_{\varepsilon}$. The next result is interpreted as a variant of (1.6). 
Lemma 1. Assume that $g$ is a $C^{2}$ function and $g \in K_{\alpha}$ for some $\alpha>0$. Let $u^{\varepsilon}$ be the solution of $\left(A_{\varepsilon}\right)$ and $\Omega$ be a smoothly open set in $R^{n}$. Suppose that $\theta$ is a nondecreasing $C^{2}$ function on $R$. Let $a^{\varepsilon}, v^{\varepsilon}, B^{\varepsilon}, E^{\varepsilon}$ be defined as (3.5)-(3.8). Then for $t_{0}, 0 \leq t_{0}<\infty$

$$
v^{\varepsilon}(0)-v^{\varepsilon}\left(t_{0}\right) \leq t_{0}^{1 / 2}\left[a^{\varepsilon}(0)^{2} / 2-a^{\varepsilon}\left(t_{0}\right)^{2} / 2+B^{\varepsilon}\left(t_{0}\right)+\varepsilon E^{\varepsilon}\left(t_{0}\right)\right]^{1 / 2} .
$$

Proof. Using the equation $\left(\mathrm{A}_{\varepsilon}\right)$ we observe that

$$
v^{\varepsilon}(0)-v^{\varepsilon}\left(t_{0}\right)=\int_{t_{0}}^{0} \int_{\Omega} \theta^{\prime} u_{t}^{\varepsilon} d x d t=\int_{0}^{t_{0}} \int_{\Omega} \theta^{\prime}\left|\nabla u^{\varepsilon}\right|_{\varepsilon} H^{\varepsilon} d x d t
$$

with $H^{\varepsilon}=-\operatorname{div}\left(N^{\varepsilon}\left(\nabla u^{\varepsilon}\right)\right)$ with $\theta^{\prime}=\theta^{\prime}\left(u^{\varepsilon}\right)$. Applying the Schwarz inequality yields

$$
v^{\varepsilon}(0)-v^{\varepsilon}\left(t_{0}\right) \leq \int_{0}^{t_{0}}\left(a^{\varepsilon}\right)^{1 / 2}\left(\int_{\Omega} \theta^{\prime}\left|\nabla u^{\varepsilon}\right|_{\varepsilon}\left(H^{\varepsilon}\right)^{2} d x\right)^{1 / 2} d t .
$$

We next calculate the time derivative of $a^{\varepsilon}(t)$ :

$$
d a^{\varepsilon} / d t=\int_{\Omega} \theta^{\prime \prime} u_{t}^{\varepsilon}\left|\nabla u^{\varepsilon}\right|_{\varepsilon} d x+\int_{\Omega} \theta^{\prime} \nabla u_{t}^{\varepsilon} \cdot N^{\varepsilon}\left(\nabla u^{\varepsilon}\right) d x=I_{1}+I_{2},
$$

where $\theta^{\prime \prime}=\theta^{\prime \prime}\left(u^{\varepsilon}\right)$. Since $U_{t}^{\varepsilon}=\theta^{\prime} u_{t}^{\varepsilon}$, integrating the second term by parts yields

$$
I_{2}=-\int_{\Omega} \theta^{\prime \prime} u_{t}^{\varepsilon} N^{\varepsilon}\left(\nabla u^{\varepsilon}\right) \cdot \nabla u^{\varepsilon} d x+\int_{\Omega} \theta^{\prime} u_{t}^{\varepsilon} H^{\varepsilon} d x+b^{\varepsilon}(t) .
$$

Note that

$$
|p|_{\varepsilon}-N^{\varepsilon}(p) \cdot p=\varepsilon^{2} /|p|_{\varepsilon}=\varepsilon^{2} /\left(\varepsilon^{2}+|p|^{2}\right)^{1 / 2} \leq \varepsilon
$$

to get

$$
\begin{aligned}
d a^{\varepsilon} / d t & =\int_{\Omega} \theta^{\prime \prime} u_{t}^{\varepsilon} \varepsilon^{2} /\left|\nabla u^{\varepsilon}\right|_{\varepsilon} d x+\int \theta^{\prime} u_{t}^{\varepsilon} H^{\varepsilon} d x+b^{\varepsilon}(t) \\
& \leq \varepsilon e^{\varepsilon}(t)-\int_{\Omega} \theta^{\prime}\left(H^{\varepsilon}\right)^{2}\left|\nabla u^{\varepsilon}\right|_{\varepsilon} d x+b^{\varepsilon}(t)
\end{aligned}
$$

since $u_{t}^{\varepsilon}=-H^{\varepsilon}\left|\nabla u^{\varepsilon}\right|_{\varepsilon}$ by $\left(\mathrm{A}_{\varepsilon}\right)$. Pluging this inequality in the right hand side of (3.9) yields

$$
\begin{aligned}
v^{\varepsilon}(0)-v^{\varepsilon}\left(t_{0}\right) & \leq \int_{0}^{t_{0}}\left(a^{\varepsilon}\right)^{1 / 2}\left(-\frac{d a^{\varepsilon}}{d t}+\varepsilon e^{\varepsilon}(t)+b^{\varepsilon}(t)\right)^{1 / 2} d t \\
& \leq\left(\int_{0}^{t_{0}} d t\right)^{1 / 2}\left(\int_{0}^{t_{0}}\left(-a^{\varepsilon} \frac{d a^{\varepsilon}}{d t}+\varepsilon a^{\varepsilon} e^{\varepsilon}+a^{\varepsilon} b^{\varepsilon}\right) d t\right)^{1 / 2}
\end{aligned}
$$


by the Schwarz inequality. Since $d\left(a^{\varepsilon}\right)^{2} / d t=2 a^{\varepsilon} d a^{\varepsilon} / d t$ this inequality completes the proof of Lemma 1 by recalling definitions of $B^{\varepsilon}$ and $E^{\varepsilon}$.

We shall choose a special $\theta$. For fixed $\delta>0$ let $\theta_{\delta}$ be a nondecreasing $C^{2}$ function on $R$ such that

$$
\theta_{\delta}(\xi)=\left\{\begin{array}{lll}
\xi-\delta & \text { for } & \xi \geq 3 \delta / 2 \\
0 & \text { for } \quad \xi \leq \delta / 2
\end{array}\right.
$$

and that $0 \leq \theta_{\delta}^{\prime} \leq 1$. For this choice of $\theta=\theta_{\delta}$ the quantities defined by (3.5)-(3.8) are denoted with subscript $\delta$, for example, $a_{\delta}^{\varepsilon}, v_{\delta}^{\varepsilon}, B_{\delta}^{\varepsilon}, E_{\delta}^{\varepsilon}$ e.t.c.

Lemma 2. Assume that $g$ is a $C^{2}$ function on $R^{n}$ and $g \in K_{\alpha}$ for some $\alpha>0$. Let $D_{0}$ be a super zero-level set of $g$ i.e., $\{g>0\}$. Assume that $\Omega$ is a ball $B^{n}(R)$ containing $D_{0}$. Let $t_{*}=t_{*}\left(D_{0}\right)$ be the extinction time defined by (1.1). Let $u^{\varepsilon}$ be the solution of $\left(A_{\varepsilon}\right)$ so that $u=\lim _{\varepsilon \downarrow 0} u_{\varepsilon}$ is the solution of $(L)$. For fixed $\delta>0$ we take $\theta_{\delta}$ as above and define $a_{\delta}^{\varepsilon}, v_{\delta}^{\varepsilon}, B_{\delta}^{\varepsilon}, E_{\delta}^{\varepsilon}$ by (3.5)-(3.8). Then

(i) $\varlimsup_{\varepsilon \downarrow 0} a_{\delta}^{\varepsilon}(t) \leq \lim _{\varepsilon \downarrow 0} \int_{D_{t}}\left|\nabla u^{\varepsilon}(t, x)\right| d x$ for $t, 0 \leq t \leq t_{*}$ where $D_{t}=\{x ; u(t, x)>0\}$.

(ii) There is a positive constant $\varepsilon_{0}=\varepsilon_{0}\left(\delta, u, t_{*}, R, n\right)$ such that

$$
B_{\delta}^{\varepsilon}\left(t_{*}\right)=v_{\delta}^{\varepsilon}\left(t_{*}\right)=0 \text { for } 0<\varepsilon<\varepsilon_{0}
$$

(iii) There is a positive constant $C_{0}=C_{0}(\delta, g, R, n)$ such that

$$
\sup _{0<\varepsilon<1} E_{\delta}^{\varepsilon}\left(t_{*}\right) \leq C_{0} t_{*}
$$

Proof. (i) By the convergence (3.4) we observe that there is a small positive constant $\varepsilon_{0}=\varepsilon_{0}\left(\delta, u, t_{*}, R, n\right)$ such that

$$
u^{\varepsilon} \leq u+\delta / 2 \quad \text { on } \quad M=\left[0, t_{*}\right] \times \bar{\Omega} \quad \text { for } \quad 0<\varepsilon<\varepsilon_{0} .
$$

Let $M_{-}$be the set of $(t, x) \in M$ with $u(t, x) \leq 0$. Clearly, $u^{\varepsilon} \leq \delta / 2$ on $M_{-}$for $\varepsilon<\varepsilon_{0}$. Since $\theta_{\delta}(\xi)=0$ for $\xi \leq \delta / 2$, this implies

$$
\theta_{\delta}^{\prime}\left(u^{\varepsilon}\right)\left|\nabla u^{\varepsilon}\right|_{\varepsilon}=0 \text { on } M_{-} \quad \text { for } 0<\varepsilon<\varepsilon_{0} .
$$


Since $D_{t}$ is always contained in $\Omega$ by the comparison principle ([ES1], [CGG1]), we now obtain, by $0 \leq \theta_{\delta}^{\prime} \leq 1$, that

$$
a_{\delta}^{\varepsilon}(t)=\int_{D_{t}} \theta_{\delta}^{\prime}\left(u^{\varepsilon}\right)\left|\nabla u^{\varepsilon}\right|_{\varepsilon} d x \leq \int_{D_{t}}\left|\nabla u^{\varepsilon}\right|_{\varepsilon} d x \leq \int_{D_{t}}\left(\varepsilon+\left|\nabla u^{\varepsilon}\right|\right) d x
$$

for $0 \leq t \leq t_{*}$ and $0<\varepsilon<\varepsilon_{0}$. Sending $\varepsilon$ to zero completes the proof of (i).

(ii) Take $\varepsilon_{0}$ as in (i). By the comparison principle we see that the lateral boundary $\Sigma=\left[0, t_{*}\right] \times \partial \Omega$ of $M$ is contained in $M_{-}$. By the definition of the extinction time, $S=\left\{t_{*}\right\} \times \Omega$ is also contained in $M_{-}$. If $0<\varepsilon<\varepsilon_{0}$, we see $u^{\varepsilon} \leq \delta / 2$ on $\Sigma$ and $S$. This implies

$$
U_{\delta}^{\varepsilon}=0 \quad \text { on } S \quad \text { and }\left(U_{\delta}^{\varepsilon}\right)_{t}=0 \text { on } \Sigma
$$

since $\theta_{\delta}(\xi)=0$ for $\xi \leq \delta / 2$. We thus obtain $v_{\delta}^{\varepsilon}\left(t_{*}\right)=0$ and $b_{\delta}^{\varepsilon}(t)=0$ for $0 \leq t \leq t_{*}$ so that $B_{\delta}^{\varepsilon}\left(t_{*}\right)=0$.

(iii) Applying the estimate (3.3) we have

$$
a_{\delta}^{\varepsilon}(t) \leq C\left(1+\|g\|_{C^{2}}\right) \mathscr{L}^{n}(\Omega) \text { for } \quad 0<\varepsilon<1 .
$$

Since $\left|\theta_{\delta}^{\prime \prime}\right|$ is bounded with a bound $c=C(\delta)$ applying (3.3) to $e_{\delta}^{\varepsilon}$ yields

$$
e_{\delta}^{\varepsilon}(t) \leq \int_{\Omega} c\left\|u_{t}\right\|_{\infty} d x \leq c C\|g\|_{C^{2}} \mathscr{L}^{n}(\Omega)
$$

We thus observe that

$$
E_{\delta}^{\varepsilon}(t) \leq t \max \left\{a_{\delta}^{\varepsilon} e_{\delta}^{\varepsilon}(t) ; 0 \leq t \leq t_{*}\right\} \leq C_{0} t \quad \text { for } \quad 0<\varepsilon<1
$$

with $C_{0}=C^{2}\|g\|_{C^{2}}\left(1+\|g\|_{C^{2}}\right) c\left(\mathscr{L}^{n}(\Omega)\right)^{2}$. This completes the proof of Lemma 2.

\section{Proof of Theorem 2}

We first prove Theorem 2 by assuming that $g$ is $C^{2}$. Let $a_{\delta}^{\varepsilon}$, $v_{\delta}^{\varepsilon}$ e.t.c. be as in Lemma 2 . Applying Lemma 1 with $t_{0}=t_{*}$ yields

$$
v_{\delta}^{\varepsilon}(0)-v_{\delta}^{\varepsilon}\left(t_{*}\right) \leq t_{*}^{1 / 2}\left[a^{\varepsilon}(0)^{2} / 2+B_{\delta}^{\varepsilon}\left(t_{*}\right)+\varepsilon E_{\delta}^{\varepsilon}\left(t_{*}\right)\right]^{1 / 2} .
$$

By Lemma 2 (ii), (iii) and (i) we send $\varepsilon \rightarrow 0$ to get

$$
\varlimsup_{\varepsilon \downarrow 0} v_{\delta}^{\varepsilon}(0) \leq\left(t_{*} / 2\right)^{1 / 2} \varlimsup_{\varepsilon \downarrow 0} a_{\delta}^{\varepsilon}(0) \leq\left(t_{*} / 2\right)^{1 / 2} \int_{D_{0}}|\nabla g| d x .
$$


Since $\theta_{\delta}(\xi)=\xi-\delta$ for $\xi \geq 3 \delta / 2$ and $0 \leq \theta_{\delta}^{\prime} \leq 1$, we see

$$
\int_{D_{0}}(g-\delta) d x \leq \int_{D_{0}} \theta_{\delta}(g) d x=\int_{\Omega} \theta_{\delta}(g) d x=v_{\delta}^{\varepsilon}(0) .
$$

Combining these two inequalities yield

$$
\int_{D_{0}} g d x \leq\left(t_{*} / 2\right)^{1 / 2} \int_{D_{0}}|\nabla g| d x+\delta \mathscr{L}^{n}\left(D_{0}\right)
$$

which yields the desired inequality in Theorem 2 since $\delta>0$ is arbitrary.

To complete the proof of Theorem 2 for arbitrary $g \in K_{\alpha}$ we need an approximation lemma.

Lemma 3. Suppose that $g \in K_{\alpha}$ for some $\alpha>0$. Then there is a sequence $\left\{g_{\varepsilon}\right\}_{\varepsilon>0}$ of $C^{\infty}$ functions such that $g_{\varepsilon} \in K_{\alpha}$ and that

(a) $g-3 \varepsilon / 2 \leq g_{\varepsilon} \leq g$

(b) $\varlimsup_{\varepsilon \downarrow 0} \int_{U}\left|\nabla g_{\varepsilon}\right| d x \leq \int_{U}|\nabla g|$ for each open set $U$ in $\boldsymbol{R}^{n}$.

Completion of the proof of Theorem 2. Let $D_{0 \varepsilon}$ be the super zero-level set of $g_{\varepsilon}$ where $g_{\varepsilon}$ is given in Lemma 3.. Applying Theorem 2 for smooth $g_{\varepsilon}$ yields

$$
\int_{D_{0 \varepsilon}} g_{\varepsilon} d x \leq\left(\frac{t_{* \varepsilon}}{2}\right)^{1 / 2} \int_{D_{0 \varepsilon}}\left|\nabla g_{\varepsilon}\right| d x
$$

where $t_{* \varepsilon}=t_{*}\left(D_{0 \varepsilon}\right)$ is the extinction time for $D_{0 \varepsilon}$. By Lemma $3(\mathrm{a})$ we see $D_{0 \varepsilon}$ is contained in $D_{0}$. This implies $t_{* \varepsilon} \leq t_{*}$ by the comparison principle. We thus obtain

$$
\int_{D_{0 \varepsilon}} g_{\varepsilon} d x \leq\left(\frac{t_{*}}{2}\right)^{1 / 2} \int_{D_{0}}\left|\nabla g_{\varepsilon}\right| d x
$$

Since $g_{\varepsilon} \rightarrow g$ uniformly by Lemma 3(a), sending $\varepsilon$ to zero yields

$$
\int_{D_{0}} g d x \leq\left(\frac{t_{*}}{2}\right)^{1 / 2} \int_{D_{0}}|\nabla g|
$$

where Lemma $3(\mathrm{~b})$ is now invoked. This completes the proof of Theorem 2.

Proof of Lemma 3. This is essentially known (cf. [Gi]); however we give a proof for completeness. We use a symmetric mollifier. Let $\rho$ be a nonnegative smooth function 
whose support is contained in $B^{n}(1)$ such that $\rho(-x)=\rho(x)$ and $\int \rho d x=1$. A symmetric mollifier $\rho_{\delta}$ is obtained by setting $\rho_{\delta}(x)=\delta^{-n} \rho(x / \delta)$. For $\varepsilon>0$ we set

$$
2 \delta_{\varepsilon}=\inf \left\{\delta ;\left\|g * \rho_{\delta}-g\right\|_{\infty} \geq \varepsilon / 2\right\} \quad \text { and } \quad g_{\varepsilon}=g * \rho_{\delta}-\varepsilon \quad \text { with } \quad \delta=\delta_{\varepsilon}
$$

Since $g \in K_{\alpha}$, we see that $g * \rho_{\delta}$ converges uniformly to $g$ in $R^{n}$ as $\delta \rightarrow 0$ so that $\delta_{\varepsilon}>0$ for $\varepsilon>0$. This guarantees that $g_{\varepsilon}$ is well-defined. The property (a) is obvious.

It remains to prove (b). Integration by parts yields

$$
\int g_{\varepsilon} \operatorname{div} \varphi d x=-\int\left(g * \nabla \rho_{\delta}\right) \cdot \varphi d x \quad \text { for } \quad \varphi \in C_{0}^{\infty}\left(U, R^{n}\right)
$$

with $\delta=\delta_{\varepsilon}$, where integrals are over $R^{n}$. Since $\rho(-x)=\rho(x)$ implies that $\left(\nabla \rho_{\delta}\right)(x)=$ $-\left(\nabla \rho_{\delta}\right)(-x)$, we observe that

$$
\begin{aligned}
-\int\left(g * \nabla \rho_{\delta}\right) \cdot \varphi d x & =\iint g(y) \nabla \rho_{\delta}(y-x) \cdot \varphi(x) d y d x \\
& =\int g(y) \operatorname{div}\left(\left(\varphi * \rho_{\delta}\right)(y)\right) d y \quad \text { with } \quad \delta=\delta_{\varepsilon} .
\end{aligned}
$$

Since $\varphi * \rho_{\delta}\left(\delta=\delta_{\varepsilon}\right)$ is compactly supported in $U$ for sufficiently small $\varepsilon>0$ and since $\left\|\varphi * \rho_{\delta}\right\|_{\infty} \leq\|\varphi\|_{\infty}$, the definition of the total variation of $\nabla g$ and $\nabla g_{\varepsilon}$ in $U$ yields (b).

\section{Miscellaneous remarks}

Our estimate (1.3) is consistent with (1.2). In fact, combining (1.2) and (1.3) yields the isoperimetric inequality

$$
\mathscr{L}^{n}\left(D_{0}\right) \leq(C / 2)^{1 / 2} \mathscr{H}^{n-1}\left(\partial D_{0}\right)^{n /(n-1)}
$$

When $n=2$, the equality in (1.3) is attained if and only if $D_{0}$ is a disk. For $n \geq 3$ the equality in (1.3) is never attained provided that $D_{0}$ is convex. indeed, the formal proof of (1.3) in Section 1 applies to both cases. To keep the equality in the proof, the mean curvature $H$ must be a constant in space valiables. By Alexandorf's theorem $[\mathrm{A}]$ such an embedded surface must be a sphere. Thus $D_{0}$ must be a ball provided that the equality in (1.3) holds. For $n=3$, the disks attain the equality in (1.3). However, for $n \leq 3$, a ball $D_{0}$ never attains the equality. This complete the proof. We believe that for $n \leq 3$ the 
equality in (1.3) attains for all smooth $D_{0}$, but we do not have the proof right now.

In [ES1] and [ES2] there are examples that the zero-level set $\Gamma_{t}$ of solution $u$ of (L) extinct instantaneously even if $\Gamma_{0}$ is non-empty. However, in all their examples there is no nontrivial bounded open set $D_{0}$ such that $\Gamma_{0}$ contains $\partial D_{0}$. So their observation does not conflict to our result (1.3).

Suppose that the inward mean curvature is positive everywhere on $\Gamma_{0}=\partial D_{0}$. Then the generalized solution $D_{t}$ of $(\mathrm{E})$ is given as

$$
D_{t}=\left\{x \in D_{0} ; U(x)>t\right\}
$$

with $U$ solving

$$
\left\{\begin{array}{ccc}
-\Delta U+\sum_{1 \leq i, j \leq n} \frac{U_{i} U_{j}}{|\nabla U|^{2}} U_{i j}=1 & \text { in } & D_{0} \\
U=0 & \text { on } & \partial D_{0}
\end{array}\right.
$$

in the viscosity sense [ES1]; here $U_{i}=\partial U / \partial x_{j}, U_{i j}=\partial^{2} U / \partial x_{i} \partial x_{j}$. This equation is formally obtained from (L) by setting $u=U(x)-t$. The extinction time $t_{*}$ is the maximum $U_{M}$ of $U$ over $D_{0}$. Our estimate (1.3) gives

$$
U_{M} \geq 2\left(\mathscr{L}^{n}\left(D_{0}\right) / \mathscr{H}^{n-1}\left(\partial D_{0}\right)\right)^{2}
$$

There are several results on a lower bound of the maximum $u_{M}$ of solution $u$ of semilinear elliptic boundary value problems (cf. [PSS] and [S]). For example if $u$ solves

$$
\left\{\begin{aligned}
-\triangle u=1 & \text { in } \quad D_{0} \\
u=0 & \text { on } \quad \partial D_{0}
\end{aligned}\right.
$$

then $u_{M} \geq 2^{-1}\left(\mathscr{L}^{2}\left(D_{0}\right) / \mathscr{H}^{1}\left(\partial D_{0}\right)\right)^{2}$ when $n=2$. This is found for example in [PSS, §5] where $u$ is replaced by $2^{-1} u$. Note that $U$ is a supersolution of this equation provided that $U$ is concave. It would be interesting to compare our result with their various estimates.

Finally, it would be interesting to extend our estimates for motion driven by anisotropic curvatures; see a book of Gurtin [Gu] and references there. Generalized solutions are constructed for such a motion in [CGG1]. Approximate level set equations are studied for such motions in [CGG2]. 


\section{References}

[A] Aleksandrov, A.D.: Uniquness theorems for surfaces in the large V, AMS Translaton 21, Ser.2, 412-416 (1962).

[AAG] Altschuler, S., Angenent, S., Giga, Y.: Mean curvature flow through singularities for surfaces of rotation, Hokkaido Univ. Preprint Series \# 130, December 1991.

[B] Brakke, K.A.: The Motion of a Surface by its Mean Curvature. Princeton, Princeton University Press (1978).

[CGG1] Chen, Y.-G., Giga, Y., Goto, S.: Uniqueness and existence of viscosity solutions of generalized mean curvature flow equations, J. Differential Geometry 33, 749-786 (1991).

[CGG2] Chen, Y.-G., Giga, Y., Goto, S.: Analysis toward snow crystal growth, Proceeding of a Symposium (Sapporo 1990), pp. 43-57. Functional Analysis and Related Topics (ed. S.Koshi), Singapore: World Scientific 1991.

[CGG3] Chen, Y.-G., Giga, Y., Goto, S.: Remarks on viscosity solutions for evolution equations, Proc. Japan Acad., 67, Ser.A, 263-266 (1991).

[ES1] Evans, L.C., Spruck, J.: Motion of level sets by mean curvature I, J. Differential Geometry 33, 635-681 (1991).

[ES2] Evans, L.C., Spruck, J.: Motion of level sets by mean' curvature II, Trans. AMS (to appear)

[ES3] Evans, L.C., Spruck, J.: Motion of level sets by mean curvature III, J. Geometric Analysis 2, 121-150 (1992).

[ES4] Evans, L.C., Spruck, J.: Motion of level sets by mean curvature IV, preprint.

[F] Federer, H.: Geometric Measure Theory. New York: Springer 1969.

[GaH] Gage, M., Hamilton, R.S.: The heat equation shrinking of convex plane curves, J. Differential Geometry 23, 69-96 (1986).

[Gi] Giusti, E.: Minimal Surfaces and Functions of Bounded Variation. Boston-BaselStuttgart: Birkhauser 1984.

[Gr1] Grayson, M.A.: The heat equation shrinking embedded plane curves to round points, J. Differential Geometry 26, 285-314 (1987).

[Gr2] Grayson, M.A.: A short note on the evolution of a surface by its mean curvature, 
Duke Math. J. 58, 555-558 (1989).

[Gu] Gurtin, M.E.: Thermomechanics of Evolving Phase Boundaries in the Plane. Oxford, Clarendon Press 1993.

[H] Huisken, G.: Flow by mean curvature of convex surfaces into sphere, J. Differential Geometry 20, 237-266 (1984).

[I] Ilmanen, T.: Convergence of the Allen-Cahn equation to Brakke's motion by mean curvature, preprint.

[PSS] Payne, L.E., Sperb, R., Stakgold, I.: On Hopf type maximum principles for convex domains, Nonlinear Analysis, TMS, 1, 547-559 (1977).

[S] Sperb, R. Maximum Principles and their Applications. New York, Academic Press 1981. 